\title{
Endophthalmitis caused by Bacillus cereus: a devastating ophthalmological emergency
}

\author{
KC Lam *
}

Hong Kong Med J 2015;21:475.e1-2

DOI: 10.12809/hkmj154526

A 79-year-old man with lymphoma was admitted for chemotherapy in November 2014. During his admission, he complained of acute onset of left eye pain with loss of vision. There was no history of previous eye disease or injury.

On examination, his left eye had no light perception and intra-ocular pressure was raised to more than $50 \mathrm{~mm} \mathrm{Hg}$. There was left eye proptosis, chemosis, and oedema over the eyelid. There was no fundal view. B-scan performed by the ophthalmologist showed increased vitreous echogenicity. A complete blood picture showed low levels of haemoglobin (104 $\mathrm{g} / \mathrm{L})$ and thrombocytopenia $\left(13 \times 10^{9} / \mathrm{L}\right)$, and total white cell count reduced to $0.69 \times 10^{9} / \mathrm{L}$. Blood was taken from the central line for culture. Computed tomographic scan of the orbit was performed to look for intra-orbital haematoma as the platelet level was low. Computed tomographic scan showed left proptosis, left eyelid swelling, and increased soft tissue stranding in the retro-ocular space (Fig 1). The lens was dislocated into the posterior chamber and the density of vitreous humour was increased when compared with the right globe. The difference in densities between the two globes was exaggerated at a follow-up scan 6 hours later (Fig 2). Overall radiological findings were compatible with severe inflammation of the globe with pus in the posterior chamber complicated by lens dislocation. The patient was diagnosed with endophthalmitis and antibiotic treatment was started. The patient began to have pustular discharge from a ruptured corneal ulcer and subsequent evisceration of the eye was required. The specimen and the initial blood culture were positive for Bacillus species. The organism was sensitive for vancomycin and gentamicin.

Endophthalmitis can be classified broadly into endogenous or exogenous and can cause permanent blindness. Exogenous endophthalmitis is usually due to trauma or postoperative infection. Endogenous endophthalmitis is commonly due to bacteraemia and immunocompromised patients are at particular risk.

Endogenous endophthalmitis is relatively uncommon, accounting for $2 \%$ to $8 \%$ of all endophthalmitis cases. Staphylococcus aureus, Bacillus cereus, Escherichia coli, Neisseria meningitidis, and Klebsiella are common pathogens. ${ }^{1}$ Bacillus cereus is a highly virulent organism as it can produce toxins that trigger severe intra-ocular inflammation and can cause complete loss of vision or destruction of the globe within 24 to 48 hours. ${ }^{1,2}$ Patients may end up with enucleation and permanent loss of vision. Intravenous drug abusers are prone to Bacillus infection. ${ }^{3}$ The cause of Bacillus infection in our patient was unknown although the central

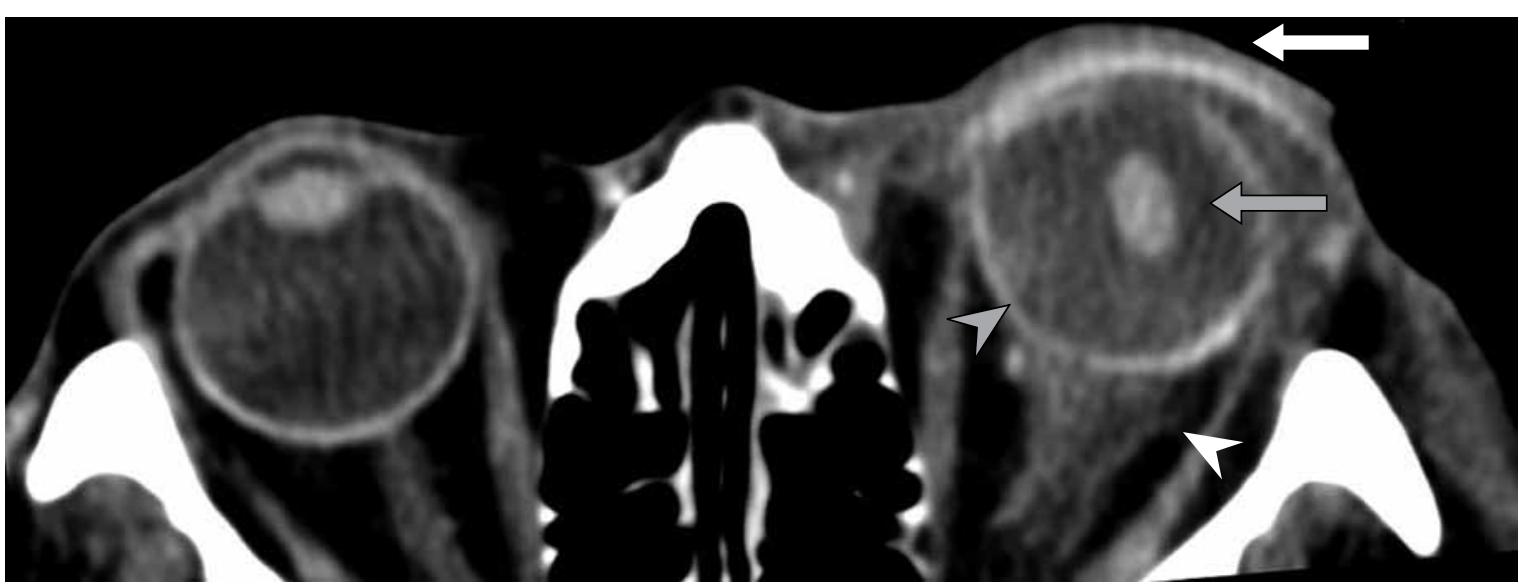

FIG I. A contrast computed tomographic scan of orbit shows left proptosis (white arrow). The lens was dislocated posteriorly (grey arrow). The vitreous humour in left globe is more hyperdense when compared with contralateral side (grey arrowhead). Increased retro-orbital soft tissue density around the optic nerve is noted (white arrowhead). There is thickening of the eyelid (white arrow) 


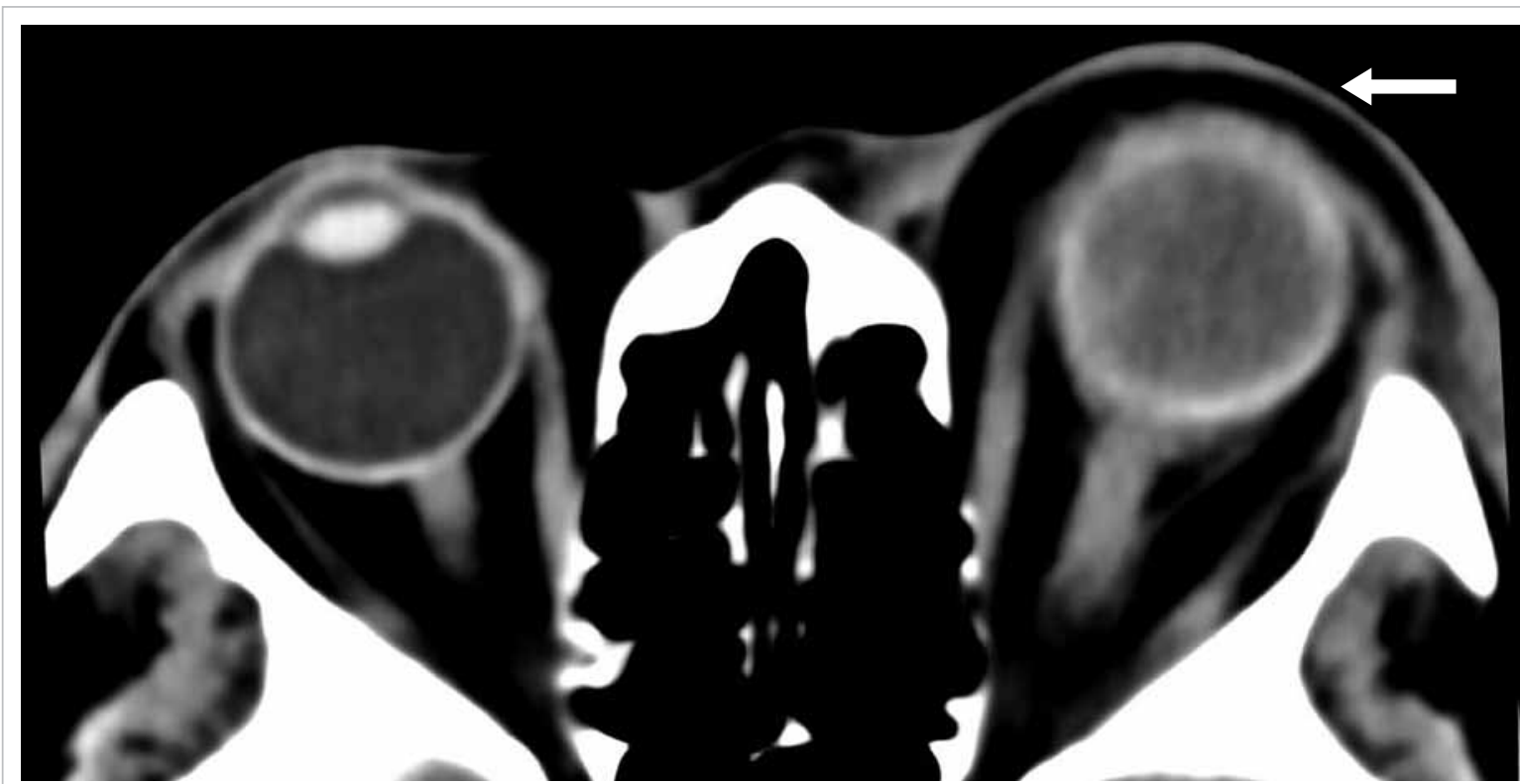

FIG 2. The difference in densities between two globes was exaggerated in follow-up scan performed 6 hours later. The lens had changed its location within the globe (not shown). Left eyelid skin thickening persisted (white arrow)

venous catheter was a potential culprit.

This article illustrates the radiological features of endophthalmitis. It is usually a clinical diagnosis although imaging may be required in complicated cases. The prognosis for Bacillus endophthalmitis is poor despite vigorous treatment.

KC Lam *, MB, BS, FRCR

Department of Radiology, Queen Mary Hospital, Pokfulam, Hong Kong

* Corresponding author: kclammbbs@gmail.com

\section{References}

1. Callegan MC, Engelbert M, Parke DW 2nd, Jett BD, Gilmore MS. Bacterial endophthalmitis: epidemiology, therapeutics, and bacterium-host interactions. Clin Microbiol Rev 2002;15:111-24.

2. Kumar N, Garg N, Kumar N, Van Wagoner N. Bacillus cereus panophthalmitis associated with injection drug use. Int J Infect Dis 2014;26:165-6.

3. Hatem G, Merritt JC, Cowan CL Jr. Bacillus cereus panophthalmitis after intravenous heroin. Ann Ophthalmol 1979;11:431-40. 